\title{
ACCESIBILIDAD AL TRANSPORTE PÚBLICO: CONDICIONES DE ACCESO: EL AUTOMOVIL
}

\author{
Diego Armando Céspedes Àlvarez ${ }^{1}$
}

Fander de Oliveira Silva ${ }^{2}$

\begin{abstract}
RESUMEN
En este artículo se procura analizar cómo el acceso al transporte público está condicionado al acceso, siendo unos de los puntos primordiales en la calidad de vida de las personas que se movilizan diariamente por los diferentes espacios. Además, se pretende descubrir cómo las políticas públicas pueden condicionar acciones para lograr avances importantes en la movilidad sustentable, asimismo cómo el acceso del transporte público puede determinar la inclusión social como medio de movilidad en el territorio Brasilero que ha sido influido en su organización territorial por el transporte privado (automóvil) en las últimas décadas, en particular este es un análisis de caso particularmente en Brasil.
\end{abstract}

PALABRAS CLAVES: Accesibilidad, Movilidad, Transporte Público, Desplazamiento, Espacios Públicos.

\section{ACESSIBILIDADE AO TRANSPORTE PÚBLICO: CONDIÇÕES AO ACCESO: O AUTOMOVEL}

\begin{abstract}
RESUMO
Este artigo procura-se analisar como o acesso ao transporte público está condicionado ao acceso, sendo os mesmo uns dos pontos primordiais na qualidade de vida das pessoas que mobilizar-se diariamente por os diferentes espaços. Além se pretende descobrir como as políticas públicas podem condicionar ações para lograr avances importantes na mobilidade sustentável, assim mesmo como o acesso do transporte público pode determinar a inclusão social como médio de mobilidade no território brasileiro que há sido influído em sua organização territorial pôr o transporte particular nas últimas décadas, particularmente no Brasil.
\end{abstract}

PALAVRAS-CHAVES: Mobilidade, Acessibilidade, Transporte Público, Espaços Públicos.

\section{ACCESSIBILITY TO PUBLIC TRANSPORT: CONDITIONS OF ACCESS: THE CAR}

\footnotetext{
${ }_{1}^{1}$ Geógrafo, Universidade Federal de Uberlândia. fanderoliveira@hotmail.com.

${ }^{2}$ Geógrafo, Universidade Nacional de Costa Rica. diegouna.geo@gmail.com. 


\section{Revista Nacional de}

Gerenciamento de Cidades

transporte público en evoluciona como el progreso de todos los otros medios de transporte y cualidades que las ciudades ofrecen a como se da sus crecimientos.

Por otro lado en Brasil a partir de la década de 1930 con el objetivo de crear un país moderno en el gobierno de Getulio Vargas con iniciativas de tener un plan viario, como discusión que se viene dando desde la primer República en atravesar el país con carreteras como un proyecto que para su entonces no se daría, pero que para 1950 con la entrada de industrias automovilísticas, llegada de la misma sucesiva de una serie de acontecimientos.

El dejar de invertir en líneas férreas e incremento acelerado de la red viaria creo que se fundaran una serie de instituciones para el control de la red y ordenamiento de lo que se pensaba que se podría dar con la entrada de las industrias automovilísticas, como parte del crecimiento económico, una cuestión que para las décadas de 1950 con la idea de gobernar para poblar pero antes se debe tener carreteras (PEREIRA, 2014).

Una tendencia del uso de automóvil que inicia desde a inicios del siglo XX, dando un acelerado crecimiento a partir de la Segunda Guerra Mundial, que para la década de 1990 se daría un crecimiento acelerado, que se mantendría hasta la época en un planeamiento urbano, traería consigo el tener las ciudades como centros de movilización en automóvil, implicando en los espacios públicos, como aceras (calzadas) usadas para estacionarse, condicionando los espacios públicos, este mismo repercutiendo en el transporte público. Estos como medio de transporte hegemónico, principalmente en Latinoamérica o bien en el territorio de Brasil, superan muchas veces la capacidad sobre la infraestructura, provocando que el espacio donde se debería tener aceras, se expandiera los anchos para estacionar automóviles o bien ampliar para que se dé mayor flujo de automóviles.

Siguiendo a Vasconcellos (2014) en Brasil las grandes ciudades y el transporte público entre los años 1960 y 2000 fueron afectados por los grandes congestionamientos producto al uso de automóviles para este periodo. Si volvemos a tiempos más recientes con el incremento acelerado de automóviles y estos 


\section{Revista Nacional de}

Gerenciamento de Cidades

efectos, se podría decir que cada día son mayores y no se ha dado un cambio notable, esto ha perturbado la velocidad de circulación de los ómnibus, repercutiendo en los tiempos y las tarifas por impactos de consumo, así como en los espacios donde se puede dar una movilidad no motorizada como a pie o en bicicleta (VASCONCELLOS, 2014, p. 155).

Entonces es aquí donde las condiciones de la economía como dice (PONS Y REYNÉS, 2014) generan que la demanda especifica de transporte en nuestros modelos de desarrollo sea por medio del uso particular, un uso privado que llegan a congestionar las principales vías de acceso a la ciudad, dejando de lado el transporte público para realizar los desplazamientos al destino deseado, influenciado por las condiciones del transporte público.

Según datos obtenidos por el Observatorio de Movilidad Urbana (2009) grafico 11, muestra como el transporte privado (automóvil) consume mucho más energía que el transporte público (ómnibus) en el caso de Sao Paulo sobre pasando el $80 \%$ del consumo de energía y contaminación, referente a un congestionamiento por altas tasas de movilidad por medio de transporte privado, pero que los mismos datos muestran como una ciudad como Curitiba donde ha realizado grandes inversiones por el transporte público el consumo sobrepasa el $60 \%$, con políticas de mayor gestión al uso del transporte público.

El congestionamiento se da en las ciudades hasta con pocos vehículos circulando uno de tras de otro, situación que no favorece la circulación sobre las vías públicas, si pensamos esto en horas pico de circulación simultánea, pasa a ser una situación absurda Vasconcellos (2014), no solo por el tiempo que se pierde y la poca agilidad al desplazarse, sino también el ocio que los usuarios dedican cotidianamente al estar dentro de un automóvil o bien un autobús, que es producto del congestionamiento afecta la calidad de vida.

Siguiendo estas rutas del hogar al trabajo, se ocasiona un movimiento cotidiano y una demanda sobre los diferentes medios de transporte, cual dinamizan las diferentes economías de las ciudades, pero a su vez produce el incremento de 


\section{Revista Nacional de}

Gerenciamento de Cidades

vehículos y que los usuarios tiendan hacer desplazamientos mayores del centro a la periferia, ocasionando efectos sobre el medio, (RAMÍREZ, 2012) destaca algo que no se puede negar; el automóvil genera ventajas económicas si se relaciona con el valor agregado, la distancia, desplazamiento, tiempo y costos, en muchos casos el automóvil trae consigo una serie de ventajas, pero desde el punto de vista individual, genera desigualdades en su uso.

Sin embargo Ramírez (2012) menciona que el crecimiento vehicular y su uso desmedido ha venido acompañado de impactos negativos sobre el ambiente, sector económico, urbano y social, reduciendo las ventajas (externalidades positivas) sobre la calidad de vida, (VASCONCELLOS, 2014, p. 163) grafica los impactos del consumo de energía y emisión de gases entre el uso del automóvil y el autobús, cual muestra datos para las ciudades principales de Brasil, el transporte privado (automóvil) es la principal fuente de contaminación y consumidor de energía muy por encima del transporte colectivo (autobús o ómnibus).

En los países en desarrollo se influencia por efectos negativos (contaminación sónica, emisión de gases e inseguridad) el consumo y uso de los vehículos privados, pero por su relación a la renta con las tarifas del transporte público, tiempos de recorrido, costos y des confort, ya sea por cantidad de personas que hacen uso del transporte al mismo tiempo en una misma línea, esto crea un motivo negativo por lo cual los usuarios buscan hacer sus desplazamientos en vehículos privados, siendo esto un tema importante que debe ser estudiado con políticas de trato al cliente para saber cómo atender las demandas, así hacen uso del transporte público y no privado, para buscar una mayor sustentabilidad.

\section{POLÍTICAS EN EL TRANSPORTE}

Muchas veces la autoridad de las políticas públicas es el origen de los impactos sobre las estructuras dentro de los distintos espacios y algunas veces por carecer de un enfoque que involucre de una manera la integralidad del transporte 


\section{Revista Nacional de}

Gerenciamento de Cidades

transporte se puede aprovechar para otros gastos familiares y disminuir las pérdidas de horas con las familias.

La ciudad de Uberlândia que por su parte, al darse cuenta de esta necesidad de garantizar la igualdad de derechos a los ciudadanos, comienza para la década de los 90s con una firma de la Ley Orgánica Municipal, el objetivo deseado fue lograr una ciudad $100 \%$ accesible para todos, desde personas con discapacidad físicas, hasta la población de menores ingresos, con la apertura de una política para dar acceso al transporte, poniéndolo a disposición para que se den los movimientos a las diferentes actividades y servicios que la ciudad brinda (UBERLÂNDIA, 2010).

La gestión en políticas públicas en los transportes con una visión integral han hecho que se garanticen obligaciones que impactan la sociedad, es aquí donde Uberlândia crea un sistema de transporte sistémico con adaptaciones desde la red de autobuses hasta la de espacios públicos, llegando a ser en Brasil para el 2009 la primer ciudad en brindar un transporte $100 \%$ accesible en términos generales, con un sistema denominado de "puerta a puerta" bajo políticas establecidas (UBERLÂNDIA, 2010), pero la accesibilidad al transporte público no es solo eso sino que va más allá, implementando políticas en búsqueda de una sostenibilidad.

Brasil por su parte cabe destacar a nivel de Latino América donde la prioridad de inversión de vías mayor mente para ómnibus, donde un estudio realizado por el Observatorio de Movilidad Urbana para América Latina (2009) grafico 9 y 10 muestra que las ciudades de mayor importancia solo Bogotá se encuentra por encima de Curitiba y Sao Paulo, pero con una carencia en la inversión de vías para que se dé la movilización del peatón y de ciclo vías, siendo esto parte de políticas que la misma ciudad de Uberlândia intenta llevar acabo para priorizar el transporte público.

\section{ACCESIBILIDAD AL TRANSPORTE}




\section{Revista Nacional de}

Gerenciamento de Cidades

Desde los términos de la geografía de los transportes la accesibilidad es la capacidad de un lugar para ser alcanzado desde diferentes puntos geográficos Pons y Reynés (2014, p. 44) es decir, que esa accesibilidad depende de la distancia y de la facilidad de desplazamiento desde el domicilio a la parada del autobús, y desde aquí hasta el destino final, incluyéndose la subida y la bajada, así como, la planificación del conjunto del itinerario". Vega Pindado (2006).

Por lo consiguiente en la integración de los modos de transporte se deben tomar en cuenta muchos aspectos: sean humanos, estructurales o donde la geografía de los transportes es necesaria para poder entender desde un pensamiento más complejo esta dinámica diaria Gauthier (1970), tanto como entender que los transportes han evolucionado para abarcar distancias más lejanas en menos tiempo, implantado por una expansión en la infraestructura de redes, generando cada vez mayores desigualdades sobre los diferentes usuarios que necesitan hacer uso del transporte.

Adicionalmente la integración de los múltiples factores deben estar involucrados a la accesibilidad del transporte público Vega Pindado (2006) por efecto, los entornos de las paradas, la información a los viajeros, el diseño de los vehículos, los sistemas de gestión del tráfico y la actitud de los profesionales en servicios; son factores que se deben tomar en cuenta en la accesibilidad al transporte público, tanto como el uso de tecnología para el acceso de una manera más rápida a información, de horarios, rutas, frecuencia, color de los buses, entre otros aspectos que engloben a un sistema de redes integrado, la accesibilidad de potencial a la movilidad de los usuarios para llegar a su destino final, generando seguridad en lla realización de los viajes.

La integración de todos los factores dentro de la accesibilidad al transporte público implica también como en el uso de otros transportes automotores individuales, citando Miralles (2002) a Harvey (1989) "un costo de transacción" que encaja en muchas veces mayores beneficios al hacer uso del automóvil desde una visión individual; es aquí por el cual la accesibilidad del transporte público debe ser 


\section{Revista Nacional de}

Gerenciamento de Cidades

abordado con sensibilidad y sentido común a través de la formación y la divulgación, para percibir los elementos que causen una mudanza en la percepción del medio de transporte por el uso colectivo Vega Pindado (2006).

Por otra parte el sistema de transporte es una conformación funcional entre las redes y gestión económica Potrykoski, Taylor (1984), siendo determinada por el movimiento de flujos, como lo menciona Pons y Reynés (2004) se liga a funciones como la accesibilidad espacial, conexión productiva y las actividades mismas, por lo que su organización económica se da por los mismos movimientos que la demanda crea al uso del transporte en la necesidad global de origen y destino.

El transporte público es claramente uno de los ejes más importantes para que se den los flujos en masa de personas en espacios dentro o fuera de un territorio, ya sea en áreas urbanas, periféricas o rurales, pero como dice una Geógrafa española en su libro ciudad y transporte, deja en claro que dentro de las dimensiones espaciales esa movilidad o desplazamiento se da efectivamente por la accesibilidad Miralles (2002) cuyos movimientos van más allá, así es como Pons y Reynés retoman a García, Rosique y Segado (1996 p. 46) e indican que las "especificas capacidades funcionales de localización espacial generan una capacidad atractiva, diferencial que pueden llegar a modificar, sustancialmente, la relación causa efecto, distancia kilometro-distancia costos, tanto en términos absolutos, cuanto relativos".

Las redes de transporte constituyen el sistema dinámico de la organización regional, de la misma forma, la infraestructura es muy importante para la emisión de los flujos económicos, sin duda, la de los transportes, pues establece una relación asociante con el territorio, Pons y Reynés (2014, p. 51).

Es aquí donde los geógrafos pensamos en los transportes como elementales para que se den las dinámicas sobre los espacios geográficos, y hacemos una intervención para analizar su integración adecuada. Un punto primordial de esto, se debe a la accesibilidad en una dimensión común que no solo es vista en cómo desplazarse a un destino en un tiempo dado, sino como hacer uso del transporte en 


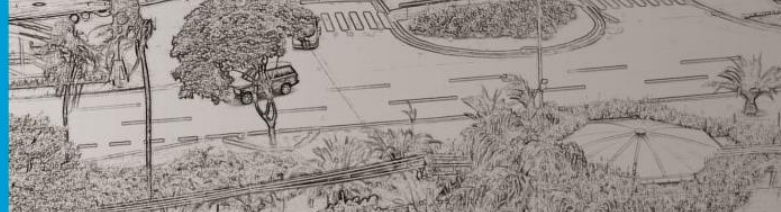

\section{Revista Nacional de}

Gerenciamento de Cidades

este caso, el público, como un medio relevante para acceder a actividades en los diversos espacios o áreas que ofrecen los servicios, siendo la movilidad cotidiana la demanda potencial de los medios de transportes Miralles (2002) esto repercute en su acceso, el cual toma en cuenta estrategias para involucrar por igual los diferentes grupos sociales en la realización de las actividades y con ello hacer el trecho de desigualdad social cada vez menor.

\section{Un transporte accesible}

La accesibilidad se encuentra dentro de un círculo que se ha desarrollado por una de las tendencias sobre el transporte como lo ha sido el alto crecimiento en las tasas de circulación de vehículo particular (automóvil), esta se toma como punto de partida en el cual la accesibilidad al transporte se ve vinculada, debido a su evolución, esta se ha dado como la misma globalización, adentrándose los espacios geográficos. Por esto podemos afirmar que se pueden analizar diferentes enfoques en el cual la geografía de los transporte se apoya en otras disciplinas.

La accesibilidad al transporte público influye sobre la organización territorial y en el bienestar de la población, la creación de estrategias organizativas y políticas puede generar que los diferentes movimientos o desplazamientos para llegar a su destino sean los cambiantes de las mismas tendencias del uso de automóvil, con la integración de mayor cantidad de espacios públicos, pero no construyendo, sino disminuyendo los flujos de vehículos que transitan sobre las mismas redes de caminos, mejorando la accesibilidad del transporte público, donde la calidad del servicio refleja en la percepción del usuario Sánchez-Flores y Romero-Torres (2010), pero antes de eso debemos analizar los usuarios y su pensar desde su punto de salida como lo es la casa a su punto destino, para este caso el uso del autobús como medio, cual debemos conocer las distintas realidades para saber cómo influenciar la población hacer uso del mismo. 


\section{Revista Nacional de}

Gerenciamento de Cidades

La estructura espacial de urbanización crea la movilidad diaria con desplazamientos generalizadas por el uso de automóvil y demandas de la población que viven en las ciudades. Esta demanda crece año con año, como causa de la industrialización en países en desarrollo donde la consolidación de centros urbanos cada vez se hace más difícil de atender desde un sentido integrado sustentable, un entendimiento que se ha dejado pasar generando consigo las desigualdades sociales.

Acontece en Uberlândia y todas las ciudades del mundo, principalmente en las latinoamericanas, que las políticas de accesibilidad en muchos de los casos no solo dejan de ser simplemente estructurales en aspectos macros, sino que dejan a un lado la opinión de los usuarios que día tras día hacen uso del sistema público, cuyo acceso debe ser eficaz y eficiente.

Se estima que para el 2015 las zonas urbanas causarán el $80 \%$ de las emisiones de $\mathrm{CO} 2$, por otra parte, los ritmos crecientes de vehículos en América Latina, han generado inseguridad vial, así como altos niveles de muertes por accidentes viales Lupano y Sánchez (2009), esta tendencia de movilidad debe ser trabajada, desde las mismas políticas en búsqueda de sistemas de transportes sustentables para que la calidad de vida sea mejorada.

La accesibilidad eficiente al transporte público no solo produce que el uso del automóvil sea disminuido, sino que las personas caminen por los diferentes sitios con mayor seguridad y tranquilidad, esto se puede ejemplificar con ciudades como Copenhague, Ámsterdam y más cercano Curitiba en el mismo territorio Brasileño. Curitiba es un ejemplo de movilidad y accesibilidad al transporte público, esta cuidad basó la movilidad de todos por medio del transporte público, y así con ello, generó que cada vez hubiese mayor cantidad de espacios públicos, incentivando para que se diesen otros desplazamientos como lo es a pie y en bicicleta. Al crear mayor accesibilidad y disminuir el uso del transporte privado se logra tener mayor espacio para la creación de ciclo vías y pasos peatonales, donde podamos caminar y desplazarnos con mayor satisfacción. 


\section{Revista Nacional de}

Gerenciamento de Cidades

Entonces, es la accesibilidad es un indicativo de movilidad, debido a que influye en la sostenibilidad y la calidad de los espacios urbanos. No se debe dejar de lado que si se quiere trabajar de puerta a puerta como el objetivo de la ciudad de Uberlândia, tenemos que tomar en cuenta muchas de las cosas que están dentro del espacio y cómo hacemos uso de él, conociendo que la accesibilidad debe empezar desde lo mínimo hasta lo más macro.

Es decir, si voy a salir de la casa pueda constar con acceso aceras (calzada) en buenas condiciones y no llenas de huecos (buracos) o como si fuese un sube y baja, es decir con una textura, anchura y estructura de las mismas condiciones, así poder llegar sin tener inconvenientes a la terminal o paradero más cercano, teniendo acceso a estas sin tener que hacer uso del automóvil.

Este tema se debe abarcar de forma inteligente, ya que el acceso y el uso del mismo están ligados a lo visual y el sentir del usuario. En los países en desarrollo se debe trabajar la información al usuario y el confort del mismo, haciendo uso de la evolución de los sistemas tecnológicos, utilizándolos como herramienta para la integración social de las personas.

Con los avances tecnológicos ya no hay marcha atrás, no se puede dejar de lado a las diferentes personas que tienen de alguna discapacidad, desde los no videntes, a quienes se les debe brindar más información de la cuidad y así generar en ellos mayor seguridad y confort. Además, se pueden implementar técnicas que beneficien a las personas que deben hacer uso de sillas de ruedas, estas técnicas consisten en con señalizaciones y pasos adecuados, estructuras que van creando una sostenibilidad social en las ciudades o centros urbanos principalmente.

La movilidad sustentable es el objetivo que todas las ciudades del mundo desean llegar y que muchas han podido a cumplir su cabalidad, pero volviendo al caso Brasilero, donde las ciudades son estructuradas por políticas de transporte pensadas en el uso del automóvil, la satisfacción colectiva se deja de lado por la circulación automovilística, esto ha producido modelos insustentables desde el punto 


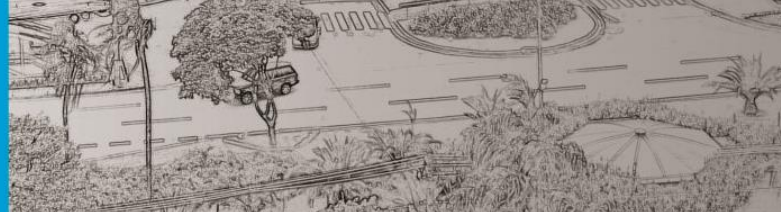

\section{Revista Nacional de}

Gerenciamento de Cidades

de vista ambiental, socioeconómico y cultural, Brasil Ministério das Cidades. (2006, p. 21).

Un planeamiento convertido por políticas públicas en pasar la populación a vivir del espacio rural a urbano (la ciudad) trajo consigo un crecimiento acelerado, colocando mayores desigualdades e exclusión social en las familias con bajos salarios, donde el transporte público debe ser el esencial servicio para la movilidad pero, con los bajos salarios que obtienen las personas llegan a gastar un alto porcentaje en pago de tarifas mensualmente como por ejemplo en Rio Janeiro se gasta hasta el 70\% del salario mínimo (Kurka, L., 2015).

Finalmente, el transporte privado trae consigo una serie de impactos negativos, como inseguridad, contaminación, estrés, mayor consumo energético, accidentes, entre otros. Todo esto afecta de diferentes maneras el medio social, económico y natural. Siendo el automóvil considerado un proceso de modernidad en Brasil Vasconcellos (2014), esto bajo un modelo político que en Brasil fue discutido desde principios del siglo XX con mayor fuerza, pero que analizando las consecuencias, estas indican todo lo contrario en relación con el transporte colectivo o público, así como no motorizado, teniendo ejemplos mundiales como Copenhague y Ámsterdam.

\section{CONCLUSIONES}

La accesibilidad al transporte público como medio de movilidad ha sido una cuestión que a nivel mundial se ha discutido para desarrollar ciudades bajo un modelo sustentable, esto principalmente en países en desarrollo los cuales han sido gravemente afectados por el uso racional del automóvil, motivados en muchos de los casos por reglamentaciones y estrategias políticas que influyen de una u otra manera sobre las dinámicas de formación territorial.

Políticas públicas que, en muchos de los casos como se observó en el estudio de caso de Brasil ha sido influenciado por modelos económicos que van más 


\section{Revista Nacional de}

Gerenciamento de Cidades

HUFFPOST VOCES. Obtenido en: http://voces.huffingtonpost.com/enrique-kogan/numero-devehiculos-en-el-mundo_b_6237052.html. Acceso en mayo 2015.

KURKA, L. Comprometimento da renda varia de $17 \%$ a $70 \%$ na região metropolitana no Rio. Notícas R7. Obtenido en: http://noticias.r7.com/rio-de-janeiro/rio-trabalhador-gasta-ate-70-de-salario-minimocom-transporte-publico-19062013. Acceso marzo 2015.

LUPANO, J. A,: Sánchez, R. J. Políticas de movilidad urbana e infraestructura urbana de transporte. Santiago de Chile: Naciones Unidas, 2009. p. 65

BRASIL. Ministério das Cidades. Mobilidade e desenvolvimento urbano. Secretaria de Transporte e da Mobilidade Urbana - Brasília: MCidades, 2006. 164p.

MIRALLES, C. Ciudad y transportes: El binomio imperfecto. Barcelona: Ariel, S. A, 2002.

OBSERVATORIO DE MOVILIDAD URBANA PARA AMÉRICA LATINA. Corporación Andina de Fomento (CAF). Información para mejores políticas mejores ciudades. Rio Janeiro, Brasil. Obtenido

en: http://www.cefid.udesc.br/arquivos/id_submenu/1131/movilidad_urbana_pp_16_11pm.pdf Acceso en mayo 2105. 2009.

PEREIRA, V de Britto. Transportes: História, crises e caminhos. Rio Janeiro: Civilizacao Brasileira. 1.ed. 322p. :il. ; 23cm. 2014

PINHEIRO, A., \& QUIRINO DA SILVA, H. As linhas do tecido urbano: O sistema de transporte e a evolução urbana de Uberlândia-MG. Uberlândia: Grafica Roma, 2006.

PONS, J. M.S.; REYNÉS, M. R.M. Geografía de los Transportes.Palma (Illes Balears): Universitat de les Illes Balears: Gráficas Planisi, S.A, 2004.

OTRYKOWSKI, M.; TAYLOS, Z. Geografía del Transporte. Barcelona: Ariel, 1984.

RAMÍREZ, S. M. La importancia de redución del uso del automóvil en México: Tendencias de motorización, del uso del automóvil y de sus impactos. México, DF: L.D.G, 2012.

SÁNCHEZ-FLORES, Ó.; Romero-Torres, J. Factores de calidad del servicio en el transporte público de pasajeros: Estudio de caso de la ciudad de Toluca, México. Economía, Sociedad y Territorio, vol. X, núm. 32, 49-80. 2010.

SANTOS, M.; Becker, B. K. Território, territórios: ensaios sobre o Ordenamiento Territorial. Rio Janeiro: DP\&A, 2006.

UBERLÂNDIA. Transporte público $100 \%$ accesible en Uberlândia: un camino a la inclusión social (Brasil). Uberlândia. Disponivel em: http://habitat.aq.upm.es/dubai/10/bp2550.html Acceso em maio 2015.

VASCONCELLOS, E. A. de. Políticas de Transporte no Brasil: a construção da mobilidade excludente. Barueri, SP: Manole Ltda. $1^{\circ} \mathrm{ed} .2014$.

VEGA PINDADO, P. (2006). La accesibilidad del transporte en autobús. Madrid: IMSERSO, 2006. 